\title{
Nickel Isotopic Composition and Nickel/Iron Ratio in the Solar Wind: Results from SOHO/CELIAS/MTOF
}

\author{
R. Karrer • P. Bochsler • C. Giammanco • F.M. Ipavich • \\ J.A. Paquette $\cdot$ P. Wurz
}

Received: 12 January 2007 / Accepted: 22 May 2007 / Published online: 3 July 2007

(C) Springer Science+Business Media B.V. 2007

\begin{abstract}
Using the Mass Time-of-Flight Spectrometer (MTOF)—part of the Charge, Elements, Isotope Analysis System (CELIAS) — onboard the Solar Heliospheric Observatory ( $\mathrm{SOHO}$ ) spacecraft, we derive the nickel isotopic composition for the isotopes with mass 58, 60 and 62 in the solar wind. In addition we measure the elemental abundance ratio of nickel to iron. We use data accumulated during ten years of SOHO operation to get sufficiently high counting statistics and compare periods of different solar wind velocities. We compare our values with the meteoritic ratios, which are believed to be a reliable reference for the solar system and also for the solar outer convective zone, since neither element is volatile and no isotopic fractionation is expected in meteorites. Meteoritic isotopic abundances agree with the terrestrial values and can thus be considered to be a reliable reference for the solar isotopic composition. The measurements show that the solar wind elemental $\mathrm{Ni} / \mathrm{Fe}$-ratio and the isotopic composition of solar wind nickel are consistent with the meteoritic values. This supports the concept that low-FIP elements are fed without relative fractionation into the solar wind. Our result also confirms the absence of substantial isotopic fractionation processes for medium and heavy ions acting in the solar wind.
\end{abstract}

Keywords Sun: solar wind · Sun: elemental composition · Sun: isotopic abundances

\section{Introduction}

The solar wind elemental composition is usually compared to the photosphere and to meteoritic elemental abundances. All three originate from the same source. However, they can differ substantially due to enrichment and fractionation processes. The solar wind is generally enriched in ions with low first ionization potential (low-FIP elements, e.g., calcium,

R. Karrer $(\bowtie) \cdot$ P. Bochsler · C. Giammanco $~ P$ P. Wurz

Physikalisches Institut, University of Bern, Bern, Switzerland

e-mail: reto.karrer@space.unibe.ch

F.M. Ipavich · J.A. Paquette

Department of Physics, University of Maryland, College Park, MD, USA 
Fig. 1 Proton Monitor. Distribution of solar wind speeds from January 1996 to January 2006. Investigated solar wind speed ranges are shaded: The speed intervals cover 290-330, $370-410$, and $560-640 \mathrm{~km} / \mathrm{s}$

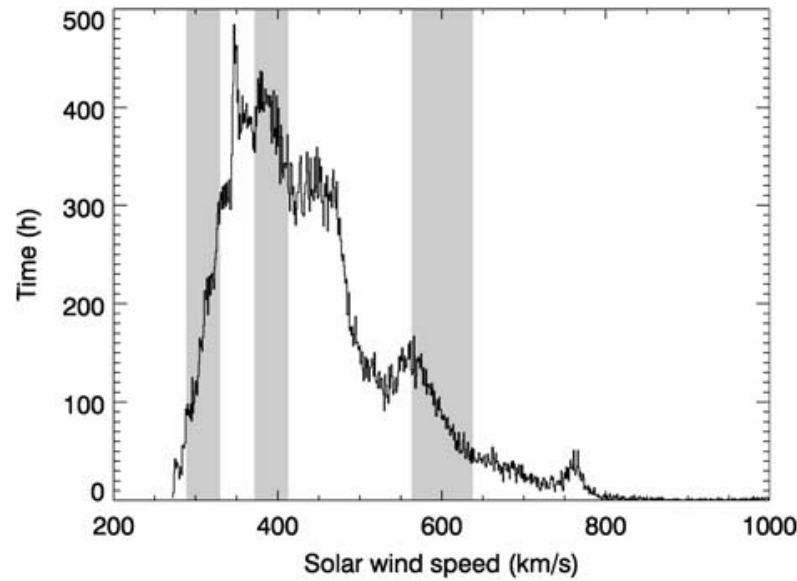

iron, nickel) compared to the photospheric abundances. If the ratio of iron to nickel agrees in the solar wind with the photosphere and with meteorites, it can be concluded that nickel undergoes the same enrichment processes as iron. Additionally, if the nickel isotopic composition agrees with meteorites, it confirms the absence of substantial isotopic fractionation processes for heavy elements in the solar wind.

We evaluated ten years' worth of SOHO/CELIAS data. We focus on slow solar wind (velocity between 290 and $330 \mathrm{~km} / \mathrm{s}$ ), on intermediate solar wind $(370-410 \mathrm{~km} / \mathrm{s}$ ) and on fast solar wind (560-640 km/s) covering the whole period between 1996-2006 (Fig. 1). The solar wind speed was measured by the Proton Monitor (PM), which is a part of MTOF (Ipavich et al. 1998).

\section{Instrumentation and Data Analysis}

The Mass Time-of-Flight Spectrometer (MTOF) is part of the Charge, Elements, Isotope Analysis System (CELIAS) onboard the Solar Heliospheric Observatory (SOHO) spacecraft (Hovestadt et al. 1995). The MTOF sensor has an excellent mass resolution, which allows us to identify rare elements and isotopes and to distinguish them from more abundant neighboring species, e.g., to resolve ${ }^{58} \mathrm{Ni}$ from ${ }^{56} \mathrm{Fe}$.

The MTOF sensor consists of an energy/charge filter (Wide-Angle Variable Energy/Charge (WAVE)) and an isochronous time-of-flight spectrometer in a V-type configuration (V-Mass). Details of the principle of operations of CELIAS/MTOF can be found in Kallenbach et al. (1997).

For each MTOF setting (WAVE electrode voltage $V_{\text {WAVE }}$ and post-acceleration voltage $V_{f}$ after WAVE) day-by-day spectra (for $400 \mathrm{~km} / \mathrm{s}$ ) or five-minute spectra (for 300 and $600 \mathrm{~km} / \mathrm{s}$ ) were aligned using different TOF offsets. To find the TOF offset value, an asymmetric fit function was applied to the spectra. The fit function is the sum of a Lorentzian and a Gaussian function centered at the ${ }^{56} \mathrm{Fe}$ peak. Then a 19-parameter function was applied to the spectra to get the raw counts of all resolved iron and nickel isotopes, using the maximum likelihood method (see Fig. 2). To maximize the likelihood the FORTRAN routine E04KDF of the NAG library was used. The shape of the asymmetric Lorentzian and the asymmetric Gaussian used for the fits are similar for all peaks. The width of an individual peak was taken to be proportional to the square root of its mass. To fit the background a linear function of the TOF was assumed. 

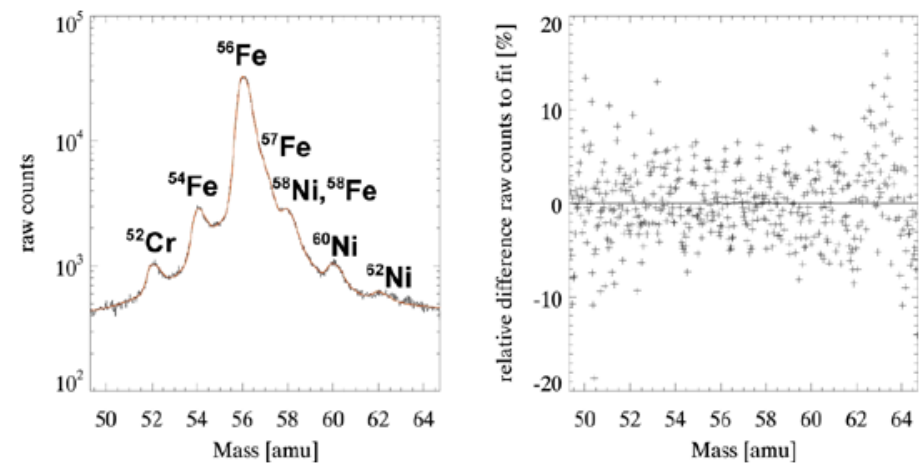

Fig. 2 Left: Accumulated MTOF spectrum from January 1996 until January 2006 for 400 km/s solar wind. $V_{f}=0 \mathrm{kV}, V_{\text {WAVE }}=12 \mathrm{kV}$. The time of flight is converted to mass, the counts are accumulated raw counts during ten years for the given MTOF settings. The peak at mass 58 comes from ${ }^{58} \mathrm{Fe}$ and ${ }^{58} \mathrm{Ni}$. This is considered in the data analysis. Right: Difference of the measured counts to the value of the 19-parameter fit function divided by the fit function value. This diagram illustrates that the fit function describes well (in the range of $\pm 10 \%$ ) the measured distribution in the mass range of 54-60 amu

To convert raw counts to particle densities, a complicated data analysis procedure is necessary (Wurz 2001). The WAVE entrance system has different transmission factors depending on the energy/charge of the entering ions. The WAVE electrode voltages were cycled in six steps covering an energy/charge range from helium to nickel. Although iron and nickel have similar kinetic energies, their charge state distributions differ (e.g., $\mathrm{Fe}^{8+}, \mathrm{Fe}^{9+}$, and $\mathrm{Fe}^{10+}$ all about $20 \%, \mathrm{Ni}^{8+}$ about $30 \%$, and $\mathrm{Ni}^{9+}$ about $50 \%$ ). For iron we used charge-state distributions observed by ACE/SWICS (cf. Gloeckler et al. 1998) in the three investigated velocity intervals to determine the transmission factors through WAVE. Since these data sets do not exist for nickel we used a simulation of the dynamic equilibrium of charge states established in the coronal expansion of the relevant solar wind velocities, which approximately reproduced the observed iron charge states. Additionally, the secondary electron yield from the carbon foil triggering the start signal of the time of flight measurement depends on the mass and the atomic number of the ion (Echenique et al. 1986). Simulations with SRIM (Ziegler 2004) indicate that the start signal efficiency is $12 \%$ lower for nickel than for iron at $1 \mathrm{keV} / \mathrm{amu}$. For the investigated peaks only the ions exiting the carbon foil with charge state +1 were counted. The fraction of singly ionized iron ions is about $5 \%$ higher than for singly ionized nickel ions at $1 \mathrm{keV} / \mathrm{amu}$ (see Gonin et al. 1995). Evaluating these effects for the three investigated velocity intervals, this results in a 10-20\% larger total detection efficiency for the dominant iron isotope ${ }^{56} \mathrm{Fe}$ relative to the dominant nickel isotope ${ }^{58} \mathrm{Ni}$. Additionally, there is a interference of ${ }^{58} \mathrm{Fe}$ to the ${ }^{58} \mathrm{Ni}$ peak. These effects are considered in the data analysis.

The differences of the three investigated nickel isotopes are small. The transmission of WAVE is optimized for ambient solar wind ions and it is therefore smaller for fast solar wind iron and nickel. This reduces the accumulated counts and increases the uncertainty of the results for $600 \mathrm{~km} / \mathrm{s}$ solar wind for nickel and iron, but it is more relevant for nickel due to lower abundances of $\mathrm{Ni}$ in the solar wind compared to Fe.

See Giammanco et al. (2006) for a detailed discussion of the instrument detection efficiency. 


\section{Results and discussion}

Tables 1 and 2 show the elemental abundances of nickel and iron and the isotopic composition of nickel. As discussed in Section 2, the detection efficiency depends both on the ion energy and on the MTOF instrument settings (WAVE electrode voltages $V_{\text {WAVE }}$ and postacceleration voltage $V_{\mathrm{f}}$ ). Thus, uncertainties given in Tables 1 and 2 are composed of varying detection efficiencies within the velocity ranges and of the different instrument settings. The presented results are within the uncertainties in good agreement with the known ratios from photosphere, solar energetic particles (SEP), earth and meteorites; see Table 1 and the references therein. Also the isotope abundance ratios for nickel agree with the known values; see Table 2 and the references therein.

Our data analysis shows that both nickel and iron experience an enrichment in the solar corona due to the lower FIP compared to the high-FIP elements (e.g., oxygen $13.618 \mathrm{eV}$ FIP, Fe $7.87 \mathrm{eV}$ and Ni $7.635 \mathrm{eV}$ ). This supports the known theories about the FIP and First Ionization Time (FIT) effects named earlier as it was previously seen for chromium (Paquette et al. 2001).

The results of this study are in excellent agreement with that presented by Ipavich et al. (2006), who also derived Ni/Fe and $\mathrm{Ni}$ isotopic abundances from MTOF, but with a different

Table $1 \mathrm{Ni} / \mathrm{Fe}$ ratios for different solar wind speeds

\begin{tabular}{lll}
\hline & Raw data & Efficiency-corrected data \\
\hline Solar wind $310 \mathrm{~km} / \mathrm{s}$ & 0.043 & $0.051 \pm 0.012$ \\
Solar wind $400 \mathrm{~km} / \mathrm{s}$ & 0.044 & $0.055 \pm 0.011$ \\
Solar wind $600 \mathrm{~km} / \mathrm{s}$ & 0.047 & $0.049 \pm 0.011$ \\
Solar wind $^{\mathrm{a}}$ & & $0.053 \pm 0.011$ \\
Photosphere $^{\mathrm{b}}$ & & $0.060 \pm 0.009$ \\
Coronal SEP $^{\mathrm{c}}$ & & $0.048 \pm 0.005$ \\
Meteorites $^{\mathrm{d}}$ & & 0.0562 \\
Earth & & 0.055 \\
\hline
\end{tabular}

${ }^{a}$ Weighted mean of the measurements above

${ }^{\mathrm{b}}$ Asplund et al. (2005)

${ }^{\mathrm{c}}$ Reames (1995)

d Lodders (2003)

Table 2 Nickel isotope abundances

\begin{tabular}{llll}
\hline & $\mathrm{Ni}^{58}$ & $\mathrm{Ni}^{60}$ & $\mathrm{Ni}^{62}$ \\
\hline Efficiency-corrected data $(310 \mathrm{~km} / \mathrm{s})$ & $0.66 \pm 0.05$ & $0.26 \pm 0.05$ & $0.08 \pm 0.04$ \\
Efficiency-corrected data $(400 \mathrm{~km} / \mathrm{s})$ & $0.75 \pm 0.07$ & $0.19 \pm 0.05$ & $0.06 \pm 0.03$ \\
Efficiency-corrected data $(600 \mathrm{~km} / \mathrm{s})$ & $0.75 \pm 0.07$ & $0.17 \pm 0.05$ & $0.08 \pm 0.04$ \\
Meteorites $^{\mathrm{a}}$ & 0.6872 & 0.2647 & 0.0367 \\
Earth $^{\mathrm{b}}$ & 0.68077 & 0.2622 & 0.03635
\end{tabular}

\footnotetext{
${ }^{a}$ Quitté et al. (2006)

${ }^{\mathrm{b}}$ Gramlich et al. (1989)
} 
approach: (a) they relied on case studies of two well-defined four-day periods, and (b) they used a different analysis technique. The agreement of the two studies provides confidence in the validity of the results.

\section{Conclusions}

This paper shows that nickel undergoes similar processes in the solar corona as other lowFIP elements, which confirms the FIP effect known from earlier works.

Acknowledgements The authors thank the entire SOHO/CELIAS team, Lisa M. Blush and Andrea Opitz for helpful suggestions. We also appreciate the helpful comments and suggestions by the reviewer. This work was supported by the Swiss National Science Foundation.

\section{References}

M. Asplund, N. Grevesse, A.J. Sauval, in ASP Conf. Ser. 336: Cosmic Abundances as Records of Stellar Evolution and Nucleosynthesis, ed. by T.G. Barnes III, F.N. Bash (2005) pp. 25-38

P.M. Echenique, R.M. Nieminen, J.C. Ashley, R.H. Ritchie, Phys. Rev. A 33(2), 897-904 (1986)

C. Giammanco, P. Bochsler, F. Ipavich, R. Karrer, J. Paquette, P. Wurz (2007), this volume. doi: 10.1007/s11214-007-9211-5.

G. Gloeckler, J. Cain, F.M. Ipavich, E.O. Tums, P. Bedini, L.A. Fisk, T.H. Zurbuchen, P. Bochsler, J. Fischer, R.F. Wimmer-Schweingruber, J. Geiss, R. Kallenbach, Space Sci. Rev. 86, 497-539 (1998)

M. Gonin, R. Kallenbach, P. Bochsler, A. Bürgi, Nucl. Instrum. Methods Phys. Res. B 101, 313-320 (1995)

J.W. Gramlich, L.A. Machlan, I.L. Barnes, P.J. Paulsen, J. Res. Nat. Inst. Stand. Technol. 94(6), 347-456 (1989)

D. Hovestadt, M. Hilchenbach, A. Bürgi, B. Klecker, P. Laeverenz, M. Scholer, H. Grünwaldt, W.I. Axford, S. Livi, E. Marsch, B. Wilken, H.P. Winterhoff, F.M. Ipavich, P. Bedini, M.A. Coplan, A.B. Galvin, G. Gloeckler, P. Bochsler, H. Balsiger, J. Fischer, J. Geiss, R. Kallenbach, P. Wurz, K.-U. Reiche, F. Gliem, D.L. Judge, H.S. Ogawa, K.C. Hsieh, E. Mobius, M.A. Lee, G.G. Managadze, M.I. Verigin, M. Neugebauer, Sol. Phys. 162, 441-481 (1995)

F.M. Ipavich, A.B. Galvin, S.E. Lasley, J.A. Paquette, S. Hefti, K.-U. Reiche, M.A. Coplan, G. Gloeckler, P. Bochsler, D. Hovestadt, H. Grünwaldt, M. Hilchenbach, F. Gliem, W.I. Axford, H. Balsiger, A. Bürgi, J. Geiss, K.C. Hsieh, R. Kallenbach, B. Klecker, M.A. Lee, G.G. Managadze, E. Marsch, E. Möbius, M. Neugebauer, M. Scholer, M.I. Verigin, B. Wilken, P. Wurz, J. Geophys. Res. 103, 17205-17214 (1998)

F.M. Ipavich, J.A. Paquette, P. Bochsler, S.E. Lasley, Proc. SOHO 17. 10 Years of SOHO and Beyond. ESA SP-617 (2006)

R. Kallenbach, F.M. Ipavich, P. Bochsler, S. Hefti, D. Hovestadt, H. Grünwaldt, M. Hilchenbach, W.I. Axford, H. Balsiger, A. Bürgi, M.A. Coplan, A.B. Galvin, J. Geiss, F. Gliem, G. Gloeckler, K.C. Hsieh, B. Klecker, M.A. Lee, S. Livi, G.G. Managadze, E. Marsch, E. Möbius, M. Neugebauer, K.-U. Reiche, M. Scholer, M.I. Verigin, B. Wilken, P. Wurz, J. Geophys. Res. 102, 26895-26904 (1997)

K. Lodders, Astrophys. J. 591, 1220-1247 (2003)

J.A. Paquette, F.M. Ipavich, S.E. Lasley, P. Bochsler, P. Wurz, in AIP Conf. Proc. 598: Joint SOHO/ACE workshop "Solar and Galactic Composition”, ed. by R.F. Wimmer-Schweingruber (2001), pp. 95-100

G. Quitté, M. Meier, C. Latkoczy, A.N. Halliday, D. Günther, Earth Planet. Sci. Lett. 242, 16-25 (2006)

D.V. Reames, Adv. Space Res. 15, 41-51 (1995)

P. Wurz, Habilitation thesis, University of Bern, Switzerland, 2001

J.F. Ziegler, Nucl. Instrum. Methods Phys. Res. B 219, 1027-1036 (2004) 